\title{
Research on the Majorization of the Internal Factor of the Incentive Mechanism of the Scientific Research and Training for Undergraduates
}

\author{
Yuan Tiebiao, ${ }^{1, *}$ \\ ${ }^{1}$ School of Information Engineering, Tianjin University Renai College, Tianjin 301636, China \\ *Corresponding author. Email: 490237091@qq.com
}

\begin{abstract} of Incentive, evaluation and feedback of incentive mechanism.

\section{INTRODUCTION}

How to cultivate high-quality innovative talents with innovative consciousness and ability has become a problem that colleges should attach great importance to and urgently need to solve. Undergraduates' participation in scientific research and training is conducive to cultivating undergraduates ' innovative spirit, improving undergraduates' innovative ability and practical ability, and comprehensively improving undergraduates' comprehensive quality. [1] Therefore, it is an effective way for undergraduates to participate in scientific research and training. Based on a questionnaire survey of 800 undergraduates in RA college, this paper makes an empirical analysis of the samples, starting with the internal factors of the incentive mechanism, and systematically studies the content of the optimization of the incentive mechanism.
\end{abstract}

In view of the current situation and existing problems of the incentive mechanism of the scientific research and training for undergraduates, through a questionnaire survey of 800 students in RA college, and taking this as a sample for empirical analysis, this paper systematically studies the internal factors of the incentive mechanism, including the subject of incentive mechanism, the object of incentive mechanism, the process of incentive mechanism, external environment and guarantee conditions of incentive mechanism, the measures

Keywords: undergraduate, scientific research and training, incentive mechanism, internal factor

\section{MAIN CONTENT}

\subsection{The subject of incentive mechanism}

The incentive subjects of scientific research and training for undergraduates include broad sense and narrow sense. In a narrow sense, the incentive subject of scientific research and training for undergraduates refers to the college and the instructor. College is the most important force to encourage undergraduate scientific research and training, including the support of scientific research funding, the construction of scientific research platform, the construction of campus culture, the construction of institutional environment. The instructor is the most direct objects to communicate with undergraduates in the process of the scientific research and training for undergraduates. The instructors play a leading role in the scientific research and training. To a great extent, the incentive effect of the instructor will affect the enthusiasm of scientific research and training for undergraduates. In broad sense, the incentive subject of scientific research and training for undergraduates divides into four aspects, including the country, the society, the school, the instructor. As shown in Figure 1, through a questionnaire survey of undergraduates in RA college, more than $90 \%$ of the undergraduates think, the most important incentive given by colleges and instructors. By comparison, the support of the country and the recognition of all sectors of society are relatively minor.

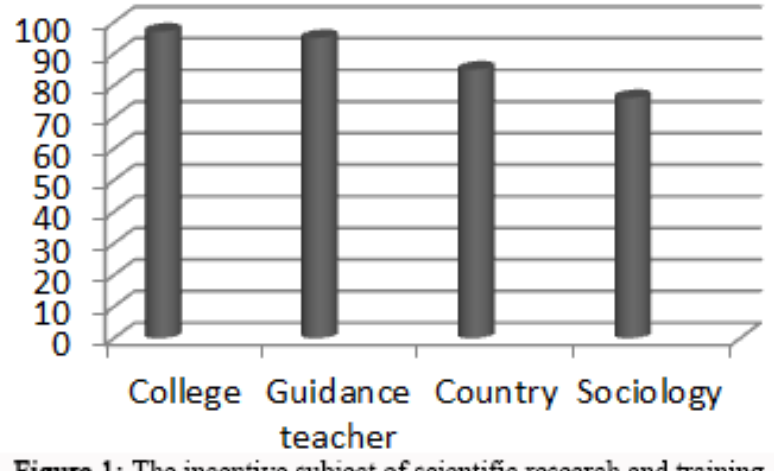

Figure 1: The incentive subject of scientific research and training for undergraduates

Therefore, it is necessary to strengthen the efforts of college to encourage scientific research and training for undergraduates and improve the ways and methods of guiding the instructor to encourage undergraduates. 
Between the college and the instructor, the role of the instructor is more important. Because scientific research and training for undergraduates is a process of two-way interaction and cooperation between instructors and undergraduates, instructors need to be aware of the necessity and significance of scientific research and training for undergraduates, in this way, undergraduates can be directly fed back in their daily guidance activities, so as to improve their enthusiasm and interest. Not only that, it is also necessary to establish a good and harmonious relationship between instructors and undergraduates to ensure smooth communication and keep full enthusiasm for scientific research.

\subsection{The object of incentive mechanism}

As shown in Figure 2, the vast majority of undergraduates realize that the incentive atmosphere within the team plays an important role in the achievement of the team's goals in the process of scientific research and training, and $41 \%$ of the undergraduates think that the overall incentive atmosphere of their team is in a strong atmosphere when they participate in scientific research and training. A strong incentive atmosphere within the team is a strong guarantee to improve team cohesion and team cooperation level. The scientific research and training for undergraduates is a research learning method of team cooperation. If a team works well, the members must have common goals, willingness to cooperate, reasonable ways of communication, and attach importance to the overall strength of the team. Only with these conditions can the cohesion and centripetal force within the team be improved and an atmosphere of mutual motivation be formed, can make every member of the team increase morale under the influence of the team culture atmosphere, can improve the overall cooperation level and enthusiasm and improve the satisfaction and sense of achievement when making progress and breakthrough.

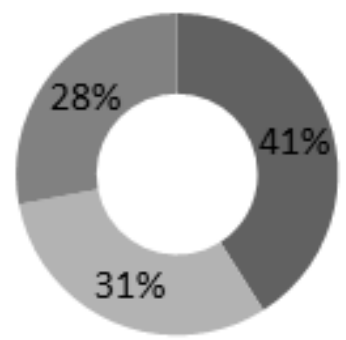

Strong

Commonly

Not strong

Figure 2: The incentive atmosphere within the team

The daily communication and learning between the project host and all team members are part of the team culture construction, which can effectively improve the incentive atmosphere within the team, in which the project host plays a very important role. When the team members are not highly motivated or lack of motivation, the project leader can take appropriate measures to motivate them, so as to mobilize the enthusiasm of the whole team. As shown in Figure 3, 85\% of the undergraduates think that the college should strengthen the overall incentive to the scientific research and training team from the outside, and as shown in Figure 4, 82\% of the undergraduates think it is very necessary to reward excellent project hosts. Additional rewards for excellent project hosts can be added on the basis of encouraging the team, so as to stimulate the enthusiasm of the host. The degree of individual incentive is strengthened that can improve the enthusiasm of each project team members. The degree of team incentive is strengthened that can improve the cooperation level and enthusiasm of the whole team, so as to further improve the enthusiasm of project members to complete scientific research and training. Therefore, the combination of team incentive and individual incentive can play a better incentive role. For the form of extra reward, credit reward and honor reward can be combined, and material reward can be given if conditions permit.

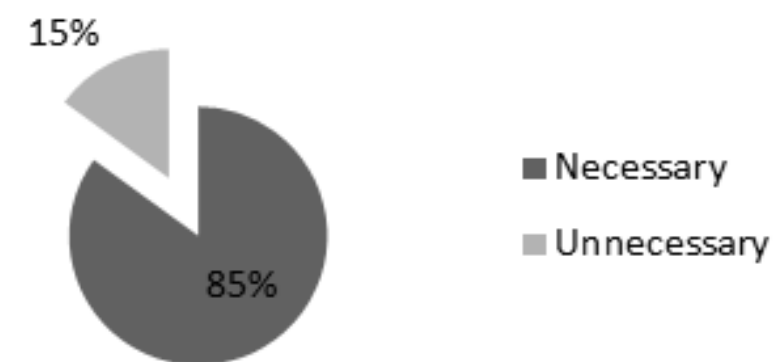

Figure 3: The necessity of the college to motivate the team as a whole

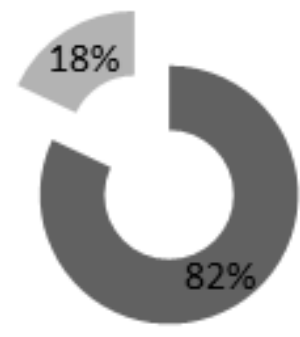

Necessary

Unnecessary

Figure 4: The necessity of rewarding excellent project hosts

\subsection{The process of incentive mechanism}

In the whole process of scientific research and training for undergraduates, we need to fully mobilize the enthusiasm of undergraduates. Every stage of motivation is essential, especially to strengthen the real-time motivation in the process of scientific research and training, because the scientific research and training for undergraduates are different from the postgraduates and the real researchers. 


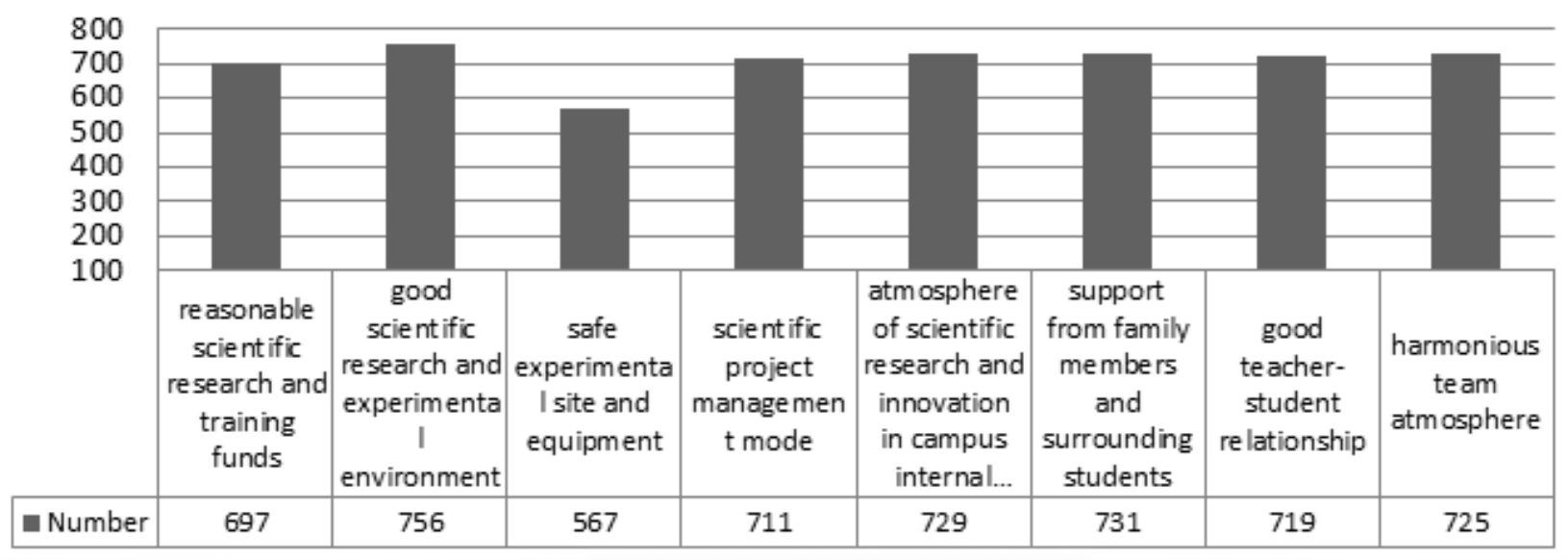

Figure 5: Selection of guarantee factors for undergraduates' enthusiasm for scientific research and training

There must be many setbacks and difficulties in the whole process of scientific research and training for undergraduates, undergraduates need more timely evaluation and encouragement from the outside, because undergraduates ' stage efforts need to be recognized, so as to improve undergraduates ' sense of achievement and satisfaction and help undergraduates to summarize the experience and lessons in the research. [2]Not only that, but also points out the direction of efforts and improvement for undergraduates, and fully mobilize the enthusiasm of undergraduates to continue scientific research and innovation in the next stage.

\subsection{External environment and guarantee conditions of incentive mechanism}

The successful operation of the incentive mechanism of scientific research and training for undergraduates can't be separated from the support of external cultural environment, system and policy environment, capital investment, hardware and equipment conditions and project management system, these provide a very important guarantee for the good operation of the incentive mechanism of scientific research and training for undergraduates.

The external factors of the incentive mechanism of scientific research and training for undergraduates include eight elements, they are reasonable scientific research and training funds, good scientific research and experimental environment, safe experimental site and equipment, scientific project management mode, atmosphere of scientific research and innovation in campus internal medicine, support from family members and surrounding students, good teacher-student relationship, harmonious team atmosphere.

As shown in Figure 5, the proportion of undergraduates choosing "safe experimental site and equipment" is relatively low. The main reason for this answer lies in the different disciplines. The experimental site and equipment of undergraduates majoring in science and engineering is provided by the college in the process of scientific research and training, [3] so they put forward higher requirements on this factor. The research methods of undergraduates majoring in humanities and social sciences are mostly investigation research, theoretical research and case study, so they don't need a fixed laboratory or equipment in the process of scientific research and training.

\subsection{The measures of Incentive}

The specific incentive measures mainly include the following aspects. (1) Undergraduates can choose their own topics and projects. (2) The college will give material rewards and commendations to excellent teams and undergraduates, and publicize excellent projects and advanced deeds of students on campus. (3) The college can provide the policy of sending postgraduates with bonus points or recommending them to be exempt from examination. (4) The college can give extra material rewards to excellent teams or reward credits of public elective courses according to comprehensive performance. (5) The college can provide students with a platform for scientific research exchange inside and outside the college or more opportunities for undergraduates to publish project results publicly. (6) Excellent achievements can be transformed into undergraduates ' graduation projects.

\subsection{Evaluation and feedback of incentive mechanism}

Evaluation refers to the objective judgment of undergraduates on the incentive effect of the college and the instructor in scientific research and training. [4]Feedback is an information transmission link that the undergraduates reflect the evaluation information to the 
undergraduates, so as to provide strong support for the college and the instructor in formulating and implementing the incentive mechanism of scientific research and training. On this basis, we can form a three-level incentive mechanism system to provide guarantee for the smooth operation and timely adjustment and improvement of incentive mechanism of undergraduate scientific research and training, they are the first is the incentive mechanism; the second is the external guarantee of the incentive mechanism; the third is the evaluation and feedback mechanism of incentive effect. This study also laid a foundation for further research on the incentive mechanism of scientific research and training for undergraduates.

\section{ACKNOWLEDGMENT}

This work was supported by Education Science Planning Project of Tianjin (HE3100).

\section{REFERENCES}

[1] Meng Jing. Research on the management system of scientific research and training for undergraduates in research universities [D]. South China University of technology, 2019

Figure 6: Undergraduates' desire to evaluate the incentive status of colleges and instructors

In the current the incentive mechanism of the scientific research and training for undergraduates, undergraduates have less opportunities to evaluate the incentive status and effect of the college and the instructor, [6] however, the questionnaire shows that most of the undergraduates are eager to get the opportunity to evaluate the college and the instructor in the process of scientific research and training or after the completion of the project (Figure 6). This survey result is highly valued by the scientific research and training management department of the college, we should fully consider the important role of students' evaluation and feedback in improving the incentive mechanism of scientific research and training and mobilizing undergraduates ' enthusiasm, and speed up the construction of the evaluation and feedback mechanism of the incentive mechanism of the scientific research and training for undergraduates.

\section{CONCLUSION}

Through in-depth research on the internal factor of the incentive mechanism of scientific research and training for undergraduates, we can fully understand the needs of
[2] Liu Yidong. Research on the subject-based undergraduate talent training mode [D]. Inner Mongolia University, 2015

[3] Bao XiuXiu. A study on the factors influencing the motivation of Teachers Guiding Undergraduate Research Projects [D]. Shanghai Normal University, 2019

[4] Gao Zhong, Liu Ji'an. From administrative control to collaborative governance: Transformation of undergraduate scientific research and training management mode $[\mathrm{J}]$. China University Science \& technology, 2019 (06): 51-54

[5] Tian Yu. Research on the management of scientific research and training for undergraduates [D]. Shanxi University, 2014

[6] Yuan Xin, sun min, Zhai musai, Tan Juncheng, Zhang Rong. Analysis and discussion of scientific research experience on the cultivation of innovation ability of Undergraduates in science and engineering [J]. Jiangsu Science \& Technology Information, 2018,35 (31): 57-59 